\title{
Application of Progressive Case Teaching Method in Visual Basic (VB) Programming Teaching
}

\author{
Jin Ling \\ College of Information Engineering, Jiangxi College of Technology, Jiangxi Nanchang 330098
}

Keywords: Progressive; Case teaching; VB programming

\begin{abstract}
Reforms were made in the teaching contents, test contents, teaching process, etc. of VB programming course; the design scheme for progressive case library and the implementation process for progressive case teaching optimized; successful implementation of teaching assured via a teaching method with vertical and horizontal layouts; and good teaching results achieved through teaching practice inspection.
\end{abstract}

\section{Introduction}

In order to improve the accomplishment of computer programming of those students whose major is not computer and the understanding of computer knowledge, many colleges and universities offer the course, VB Programming for such students. However, their weak computer fundamentals make both the teacher and students have a certain difficulty during VB teaching, and the teaching result is not ideal.

The article brings out a new case teaching method, progressive case teaching by using the experience of these teachers' thinking for reference and in combination of many years of teaching practice. Reforms are made and class, teaching and teaching plan designed, in teaching methods and cases. This new method is introduced into teaching. The result of a comparison test conducted in civil engineering and project management majors of Jiangxi University of Technology shows that using this method can greatly improve the teaching result of VB programming course.

\section{Progressive case teaching method}

This method, still based on the traditional case teaching method, is characterized by focusing on students' participation and experience and on a from-shallow-to-deep epistemology, associating cases with interesting points, considering interestingness and hierarchy, and at the same time combining cases with professional contents, enhancing the relation among all cases and avoiding use of individual cases.

Active cases can stimulate the desire hidden in students to learn and change disorderly knowledge into thinking-inspired cases. Therefore, the design of cases is critical and the basics to complete teaching. During the building of a case system, the article summarizes four principles:

(1) Take interest as a start point and courage as driving force;

(2) Replace boring concepts with programs and games;

(3) Arouse learning desire by using professional cases;

(4) Build a further case library in a progressive manner.

The cases used in the progressive case teaching method are divided into basic case, further case and systematical case, by which to build a progressive case library. Gradually improve the study effect of 
students via studying further cases from shallow to deep, gradually enhance the understanding of programming intension and make them grasp programming methods and concepts, realizing the cultivation and improvement of computer thinking.

The basic case mainly introduces basic programming concepts and grammar rules.

The further case is description of knowledge points such as three programming structures, array, process, etc., providing the students with higher requirements in flexible application and knowledge integration. The systematical case is about the comprehensive application of document and database knowledge, and is for those students having excessive learning capacity, focusing on training their innovative thinking awareness and ability.

According to the four principles for building cases, we have designed a complete teaching implementation process by using the progressive case teaching method. We divide a teaching process into longitudinal and lateral main lines. The longitudinal implementation process can be divided into three stages in combination of hierarchical case library, and each stage corresponds to different test tasks. The lateral implementation process mainly transits from teaching in class to the one out of class, and is descried in detail as follows based on the progressive case teaching scheme design:

Play a hierarchical training role in combination of a progressive case library. At the first stage, i.e. the basic case stage ( 3 - 4 weeks), have test contents interested in terms of problems such as too many concepts, where to start from, and integrate grammar rules and algorithm thinking into interesting tests so as to make students grasp the basic programming thinking of from top to bottom and stepwise refinement and form a good programming habit through such tests.

At the second stage, i.e. the further case stage (7 - 8 weeks), after grasping contents such as attribute, event, sequential structure, continue to enhance the direction and continuity of test items; and upgrade the basic validation tests at the previous stage to design tests, add test items such as code debugging, organization and testing. By these methods, most of the students' cooperation learning awareness is activated; the communication and coordination among students becomes frequent. They begin discussing whether codes can be simplified during a test process, whether the function of a program can be added, etc. At this stage, the self-exploration and coordination abilities of the students are improved to a certain level and they have the computer thinking awareness of reforming and extending cases.

At the third stage, i.e. the systematical case stage ( 4 - 5 weeks), with the programming knowledge as a support, integrate test contents such as module, multi-form, database, etc. to carry out innovative tests. During such process, the tests cannot be completed by a single student due to increased difficulty; students must cooperate with each other and carry out a large amount of study out of class under the guide of the teacher. At this stage, the emphasis is creating the innovative and application abilities of those students with excessive learning ability.

Change the dilemma with verification tests by means of adding design and innovative tests to make students gradually enhance the understanding of programming knowledge in a progressive manner, and also to make the students at different levels obtain knowledge, thus improving the programming thinking, method and ability.

\section{Form interaction in and out of class by using diversified teaching organization methods.}

1) Grouping cooperation study in class: Because of a lot of teaching contents and students, the teacher cannot teach everything in a test class, reasonable teaching grouping is a necessary to improve 
the teaching effect. Make heterogeneous grouping of students according to their learning habit and ability with 6 - 8 students for one group, with one selected for the group as the group leader, and one as the recorder. The group leader is in charge of assisting the teacher in completing test contents and solving basic problems (such as incorrect object name and sentence) met during programming; the recorder records the difficulties met during a group activity using a group activity sheet and the ways to overcome such difficulties, who are most outstanding in which aspects, and the outstanding points. Then, the teacher analyzes the deficiencies in the group activity based on such records and makes modification and adjustment to subsequent grouping strategies.

2) Stepwise task out of class: After completing each stage of learning, assign comprehensive tasks to students; and then the students in group collect information and jointly complete coding, debugging, etc. After that, record the work assigned to each group member, and prepares a discussion report. Finally the teacher randomly selects one student to discuss and elaborate the report. After a period of practice, the students have a good grasping of the whole process of programming.

3) Various forms of activities out of class: It is an effective way to give a play to the abilities of the students through various kinds of activities out of class, such as project drill, course design, VB language competition in a campus, etc. Form teaching interaction in class and out of class for the transition of teaching in class to the one out of class, cultivate the learning feeling of the students, and arouse their active awareness of learning and the potential for obtaining knowledge actively.

\section{Effect of comparison teaching}

During the teaching reform test, the progressive case teaching method was used in pilot classes (Classes 1 and 5 of Civil Engineering entered the college in 2012, and Classes 3 and 4 of Engineering Management of 2012), while the traditional teaching method in comparison classes (Classes 3 and 4 of Civil Engineering of 2012, and Classes 1 and 2 of Engineering Management of 2012). After doing so for half a year, objective analysis was made over the final exam results of the two kinds of classes, and the result shows, as indicated in Figure 1, that the exam result of pilot classes either in the Civil Engineering or in Engineering Management is higher than non-pilot classes, further indicating that the reform of the progressive case teaching really has a noticeable promotion effect on learning effect.

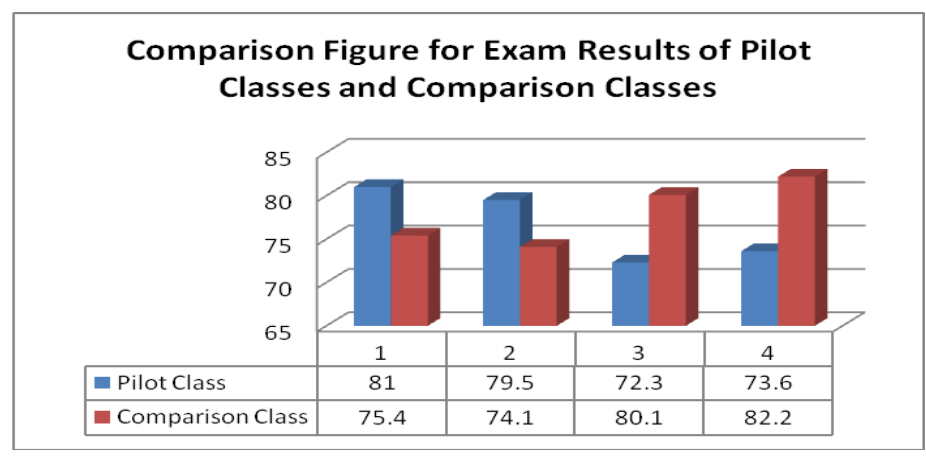

Figure. 1 Comparison Figure for Exam Results of Pilot Classes and Comparison Classes

After detailed processing of the exam results, we calculated the average of all kinds of questions in the pilot classes as indicated in Figure 2. From the figure, we can see that there are no obvious differences between choice and fill-in-the-blank questions, showing that the grasping of basic concepts and 
grammar rules by the students is similar. However, because the progressive case teaching is adopted to deepen the students' understanding of algorithm logic, code orderliness and programming functionality, the exam results of program reading and programming are noticeably higher than the comparison classes, indicating that the progressive case teaching method has a significant effect on improving the actual application ability of students.

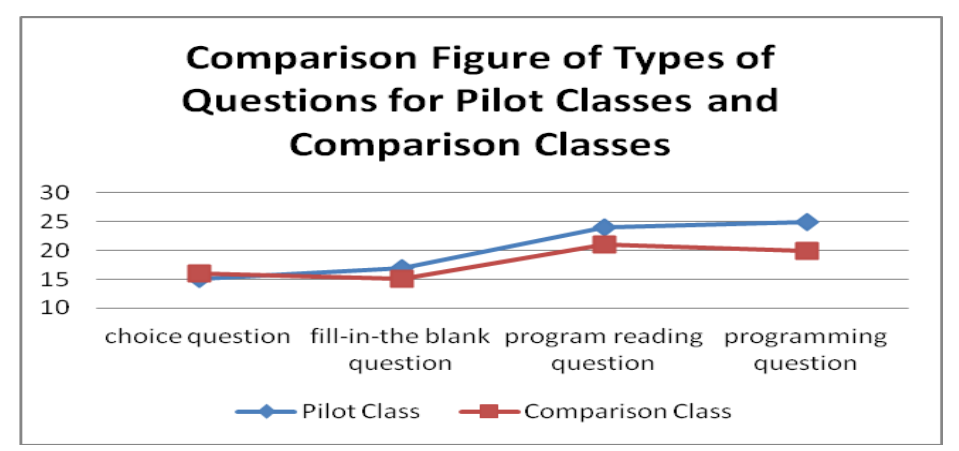

Figure. 2 Comparison Figure of Types of Questions for Pilot Classes and Comparison Classes

\section{Acknowledgment}

This article is about the application of the subject, Progressive Case Teaching in VB Programming (Subject No.: JXJG-12-19-5) studied in education and teaching reforms in Jiangxi, and the study results of the excellent course, Fundamentals of Computers in Jiangxi

\section{References}

[1] Yuan Yuan Teaching Design Study and Practice of VB Loop Structure Programming, Research of Mathematic Teaching-Learning [J], 2012 (01) : 55-57.

[2] Liu Shengtian, Qiu Haojie, Course Case Teaching Study and Teaching Resources Construction of Visual Basic Programming [J], China Adult Education 2012 (2) :145-146.

[3] Wu Haizhen, Jiang Jiafu, Application of Associated Case Teaching Method in VB Programming Teaching [J], Computer Education , 2009 (6) : 52-54. 